\title{
Complete En Bloc Urinary Exenteration for Synchronous Multicentric Transitional Cell Carcinoma with Sarcomatoid Features in a Hemodialysis Patient
}

\author{
Tiberio M. Siqueira Jr, Evandro Falcao, Tiberio M. Siqueira
}

Memorial Sao Jose Hospital, Recife, Pernambuco, Brazil

\begin{abstract}
The incidence of transitional cell carcinoma (TCC) in patients submitted to hemodialysis is low. The presence of TCC with sarcomatoid features in this cohort is even scarcer. Herein, we describe a very rare case of synchronous multicentric muscle invasive bladder carcinoma with prostate invasion in a hemodialysis patient, submitted to complete en bloc urinary exenteration.
\end{abstract}

Key words: hemodialysis; urologic neoplasms; carcinosarcoma; bladder; surgery

Int Braz J Urol. 2006; 32: 560-2

\section{CASE REPORT}

A 58 years-old white obese man, submitted to a hemodialysis program due to diabetic nephropathy, was referred to our department because of painless macroscopic hematuria.

Ultrasound and cystoscopy revealed multiple pediculated and sessile lesions in different parts of the bladder. Pre-operative computed tomography (CT) scan showed no extravesical dissemination or node enlargement.

The patient was submitted to a deep transurethral resection of bladder tumor (TURb) in part of the visible masses, but not all due to its extent. Pathologic examination showed high-grade urothelial tumor, with invasion of the lamina propria layer only.

Based on the pathological report, the patient was submitted to en bloc urinary exenteration associated to extended pelvic lymphadenectomy (Figure1). Operative time, blood loss and hospital stay were
300 minutes, $770 \mathrm{~mL}$ and 5 days, respectively. No complications were observed.

Final pathology evidenced high-grade muscle invasive bladder carcinoma with extravesical invasion to adipose tissue and bladder neck. Prostate invasion by the urothelial tumor was observed, presenting sarcomatoid features (Figure-2). Surgical margins, kidneys, ureters and nodes were free of tumor. No chemoteraphy treatment was administered.

On postoperative month 6, CT scan showed multiple lesions in both lungs and brain. Pleural biopsy evidenced bladder tumor metastasis. Six days later, the patient died of metastasis complications.

\section{COMMENTS}

It is well known that uremic patients have a higher risk to develop cancer, in which the most common types are the urological ones (1). 


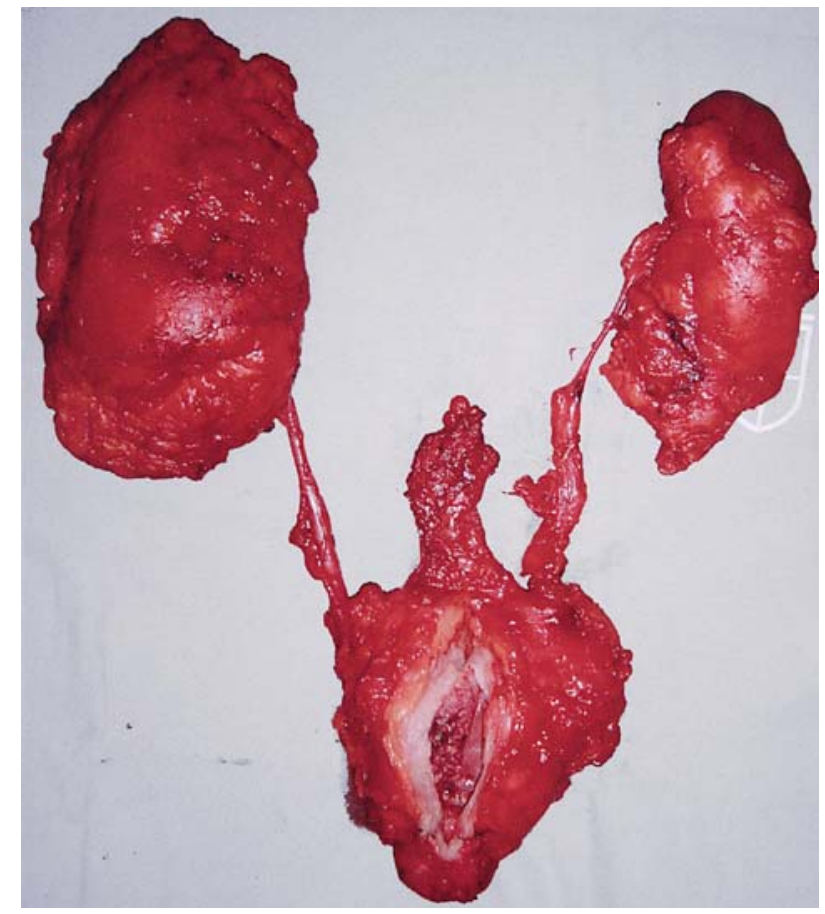

Figure 1 - Surgical specimen shows the complete en bloc removal of the entire urinary tract and prostate, associated to extended pelvic lymphadenectomy.

Recently, Wu et al. (2) reported the largest experience ever published with hemodialysis patients presenting TCC. In all 30 cases, the initial diagnosis presented gross hematuria as a cause. According to final surgical status, the patients were divided in 6 different groups. Groups 1 and 2 had total urinary exenteration (11 cases). Groups 3 and 4 had uni or bilateral nephroureterectomy with bladder cuff excision (13 cases). Group 5 had only TURb and group 6 with one case of radical cystectomy and ileal conduit.

The authors observed $100 \%$ incidence of recurrence rate in the patients of group 2, leading to a stepwise total exenteration. Likewise, the recurrence rate in groups 3,4 and 5 were 2.3, 1.7 and 1.8 times, respectively. Finally, the treatment administered to patients in group 6 was considered not ideal, due to the inability in monitoring recurrences. The conclusion was that no matter what the tumor grade, stage or location, the treatment of choice for hemodialysis patients presenting TCC is total urinary exenteration. In our case, every definitive forms of treatment were discussed with the patient. Besides that, the low residual urinary output led us choose the total "en bloc" urinary exenteration. For our surprise, final pathology showed a MIBC with sarcomatoid features and prostate invasion.

No chemotherapy was prescribed, based on the final pathological report and the patient's clinical status. The rapid progression to lungs and brain metastasis, show the aggressiveness of the tumor and made us wonder if chemotherapy would avoid this final course.

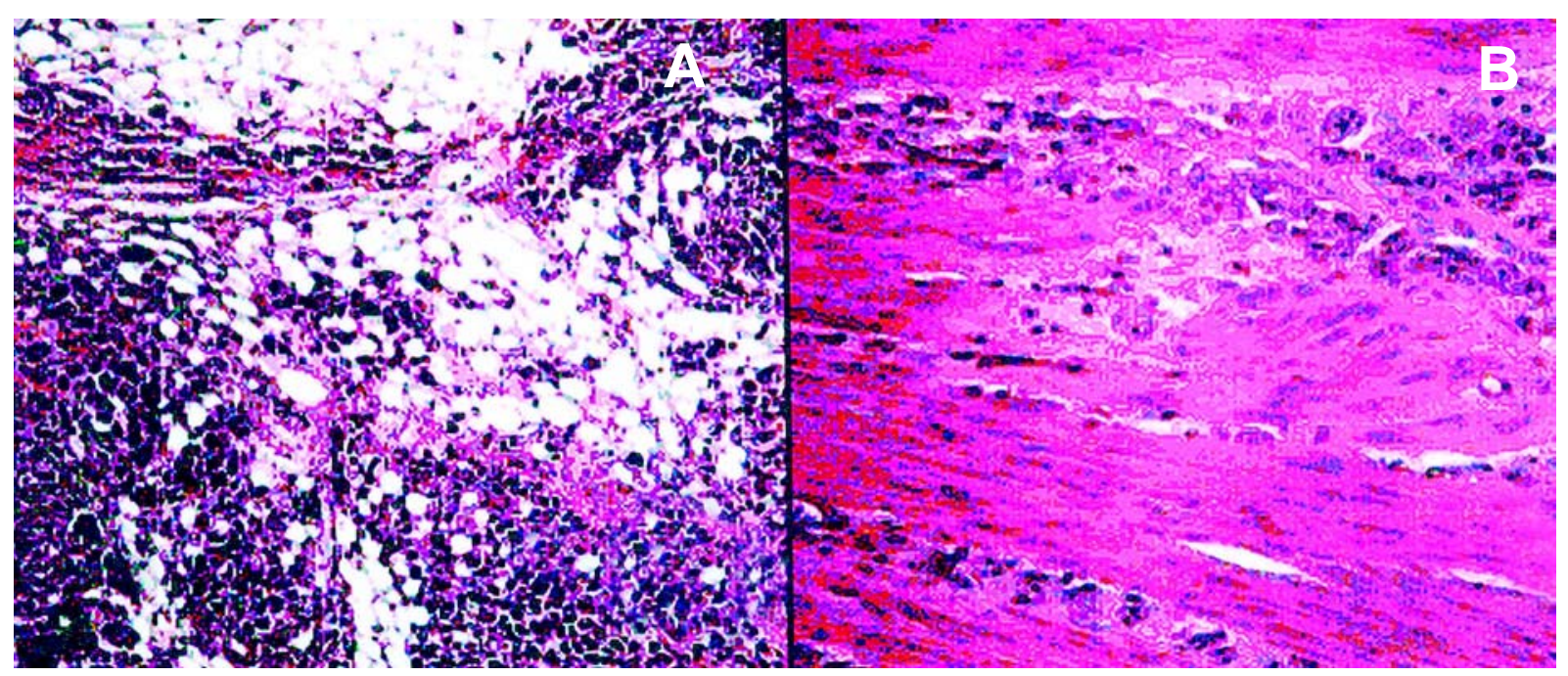

Figure 2 - Pathologic analysis. A) High grade muscle invasive bladder carcinoma with extravesical invasion to adipose tissue and bladder neck. B) Prostate invasion by urothelial tumor presenting sarcomatoid features (HE, X133). 
This case corroborates with the need to evaluate every patient in hemodialysis presenting hematuria in order to diagnose TCC. To the best of our knowledge, this is the first report of synchronous multicentric MIBC with sarcomatoid features and prostatic invasion that is treated by total "en bloc" urinary exenteration. Depending on clinical status, chemotherapy should be considered.

\section{ACKNOWLEDGEMENT}

To Dr. Adonis Carvalho for pathological examination and manuscript review.

\section{CONFLICT OF INTEREST}

None declared.

\section{REFERENCES}

1. Giacchino F, Formica M, Quarello F, Bonello F, Piccoli G: High incidence of cancer in uremic patients. Clin Nephrol. 1985; 23: 265-6.

2. Wu CF, Shee JJ, Ho DR, Chen WC, Chen CS: Different treatment strategies for end stage renal disease in patients with transitional cell carcinoma. J Urol. 2004; 171: 126-9.

\section{Correspondence address:}

Dr. Tibério M Siqueira Jr

Av. Agamenon Magalhães, 4775 / 201

Recife, PE, 50070-160, Brazil

E-mail: tiberiojr@uol.com.br 